\title{
Nonoperative management of splenic injury in closely monitored patients with reduced consciousness is safe and feasible
}

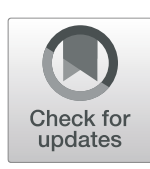

Michel Teuben ${ }^{1,2^{*}}$, Roy Spijkerman ${ }^{1}$, Taco Blokhuis ${ }^{1}$, Roman Pfeifer ${ }^{2}$, Henrik Teuber ${ }^{2}$, Hans-Christoph Pape ${ }^{2}$ and Luke Leenen ${ }^{1}$

\begin{abstract}
Background: Treatment of blunt splenic injury has changed over the past decades. Nonoperative management (NOM) is the treatment of choice. Adequate patient selection is a prerequisite for successful NOM. Impaired mental status is considered as a relative contra indication for NOM. However, the impact of altered consciousness in wellequipped trauma institutes is unclear. We hypothesized that impaired mental status does not affect outcome in patients with splenic trauma.

Methods: Our prospectively composed trauma database was used and adult patients with blunt splenic injury were included during a 14-year time period. Treatment guidelines remained unaltered over time. Patients were grouped based on the presence (Group GCS: < 14) or absence (Group GCS: 14-15) of impaired mental status. Outcome was compared.

Results: A total of 161 patients were included, of whom 82 were selected for NOM. 36\% of patients had a GCSscore < $14(N=20)$. The median GCS-score in patients with reduced consciousness was 9 (range 6-12). Groups were comparable except for significantly higher injury severity scores in the impaired mental status group (19 vs. 17, $p=$ 0.007). Length of stay ( 28 vs. 9 days, $p<0.001$ ) and ICU-stay ( 8 vs. 0 days, $p=0.005$ ) were longer in patients with decreased GCS-scores. Failure of NOM, total splenectomy rates, complications and mortality did not differ between both study groups.

Conclusion: This study shows that NOM for blunt splenic trauma is a viable treatment modality in well-equipped institutions, regardless of the patients mental status. However, the presence of neurologic impairment is associated with prolonged ICU-stay and hospitalization. We recommend, in institutions with adequate monitoring facilities, to attempt nonoperative management for blunt splenic injury, in all hemodynamically stable patients without hollow organ injuries, also in the case of reduced consciousness.
\end{abstract}

Keywords: Nonoperative management, Blunt spleen trauma, Altered mental status, GCS-score, Outcome

\section{Background}

The spleen is the most frequently injured intraabdominal organ [1,2]. Treatment of blunt splenic injury has undergone an extensive change over the past decades [3-5]. Currently, selective nonoperative management (NOM) is the preferred treatment and is used in up to $70 \%$ of patients [6-9]. A large number of

\footnotetext{
*Correspondence: michel.teuben@usz.ch

'Department of Trauma, University Medical Centre Utrecht, Heidelberglaan

100, 3584CX, Utrecht Suite G04.232, The Netherlands

${ }^{2}$ Department of Traumatology, University Hospital Zurich, Zurich, Switzerland
}

studies have validated this treatment modality with success rates exceeding $80 \%[8,10,11]$. The most important prerequisite for successful NOM is adequate patient selection. Criteria for nonoperative management have expanded with increasing experience. Nowadays, the presence of hemodynamic instability is considered the only absolute contraindication for NOM [3]. However, controversy exists regarding the impact of altered mental status on outcome of NOM. Impaired mental status is a frequent problem in patients with suspected blunt splenic injury. An impairment of mental status in

(C) The Author(s). 2019 Open Access This article is distributed under the terms of the Creative Commons Attribution 4.0 International License (http://creativecommons.org/licenses/by/4.0/), which permits unrestricted use, distribution, and 
trauma patients is most frequently a result of shock or associated head injury. Other possible causes can be intoxication, hypoxemia, hypercarbia or a combination thereof. According to Iiterature, up to $70 \%$ of patients sustaining blunt abdominal injury sustain some degree of head injury $[12,13]$.

In some institutions a altered consciousness was an absolute contraindication for observational therapy in the beginning of the NOM era. lt is believed that these patients were not suitable candidates for NOM due to the inability to perform an adequate physical examination. Unreliable serial physical examination could result in missed onset of hemorrhage, and therefore delayed surgical intervention [14-16]. The last studies investigating outcome and safety of nonoperative management of blunt splenic injury in patients with altered mental status were published more than a decade ago with treatment and monitoring options having improved markedly since then $[8,17]$. Furthermore, a recent literature review suggests that lower Glasgow Coma Scale (GCS)scores are predictive for failure of NOM [18]. In our experience, improvements in diagnostics and monitoring led to improved quality and outcome of nonoperative therapy for patients with blunt abdominal injuries. Therefore, in line with current World Society of Emergency Surgery-guidelines we do not consider a GCSscore $<12$ alone as a contraindication for NOM [19].

Therefore the purpose of this study was to determine whether the presence of neurological impairment affects the outcome of nonoperative management for blunt splenic injury in adult patients when monitored closely. We hypothesized that altered mental status in patients treated by nonoperative management for blunt splenic trauma is not associated with impaired outcome.

\section{Patients and methods}

For this study our prospectively maintained trauma database was utilized. All adult patients (age $>15$ years) who underwent therapy for blunt splenic injury during a 14year period were identified. The trauma database included all patients who were admitted to our level-Itrauma centre between January 2000 and February 2014. Due to pre-hospital triage systems in our region, severely injured patients are primarily transported to our institution. In our traumaregion, stable patients without signs of craniocerebral injuries are preferably admitted to level-II-trauma centres and therefore excluded from this analysis. Patients transferred from other facilities were excluded as well.

For the purpose of this study we included all patients that were initially selected for nonoperative management. Patients who died before total diagnostic work-up was completed, as well as patients with non-survivable brain injury were excluded.
The data gathered for each patient included: demographics, mechanism of injury (MOI), Injury Severity Score (ISS), Abbreviated Injury Score of splenic injury (AIS-spleen), Glasgow coma scale, hemodynamics on admission, and outcome. Outcome analysis included mortality, failure of NOM (fNOM), complications, length of hospital stay, and intensive care unit (ICU)-days. Splenic injuries were graded according to the Abbreviated lnjury Score, which was based on computed tomography (CT) data [20].

In all hemodynamically stable patients abdominal sonography or CT scanning with intravenous contrast was performed before they were admitted to the ICU or ward.

We defined failure of NOM as the need for laparotomy in patients initially admitted to the ICU or ward for nonoperative treatment.

Nonoperative management in our institution includes initial observation on a monitored intermediate care unit or an intensive care unit, frequent examinations of vital signs and physical examinations (including abdominal and neurological examination, fluid administration and frequent hemoglobin level measurements). The aim of intravenous fluid therapy in all patients (including those with craniocerebral injury) is to maintain systolic blood pressure of at least $90 \mathrm{mmHg}$. This semi-restrictive volume policy is believed to minimize intra-abdominal hypertension and subsequent bleeding, while maintaining sufficient organ perfusion. Angio-embolization is only indicated in patients initially selected for NOM and with deteriorating hemodynamic status due to diagnosed ongoing splenic blood loss. Our protocol including restricted utilization of angio-embolization in blunt splenic trauma has previously been shown not to be associated with impaired outcome [21]. Treatment guidelines and selection criteria for non-operative management/surgical intervention/angio-embolization did not change during the study period. Decision making was performed by the attending trauma surgeon.

Patients were divided into two groups based on presence, or absence of neurological impairment. Neurological impairment was defined as GCS-score $\leq 13$. Group I therefore consisted of patients with GCS-scores 14 and 15, and group II included patients with altered mental status (GCS-score $\leq 13$ ). GCS-score was measured for all patients upon admission. We compared morbidity and mortality between groups to determine the impact of neurological status on outcome of NOM. Complications were scored according to the ClavienDindo classification [22].

All procedures performed in this study are in accordance with the ethical standards of the institutional research committee and with the 1964 Helsinki 
declaration and its later amendments. Execution of the study was approved by the institutional review board.

All statistical analyses were performed using a commercially available statistics software package (SPSS, Version 22.0, Chicago,IL). Continuous data are expressed as median (Interquartile range) and analyzed by the Mann Whitney $\mathrm{U}$ Test. Categorical data were analyzed by the Fisher's exact test. A significance Ievel of $p<0.05$ was maintained.

\section{Results}

During the study period, a total of 161 trauma patients with blunt splenic trauma were admitted to our level-onetrauma center. The median age of our population was 32 (22-53) years, with a male predominance of $76 \%(122$ male vs. 39 female). The median ISS (IQR) was 25 (1634 ), and $64 \%$ of patients had an GCS $>13$.

As shown in Fig. 1, a total of 82 patients were treated nonoperatively for blunt splenic injury. The group consisted of 65 male and 17 female patients with a median age of 29 (range 21-51) years.

The mechanisms of injury were motor vehicle accident in 19 patients, bicycle accidents in 14 patients, motorcycle accident in 22 patients, automobile-pedestrian accident in $7 \mathrm{pa}-$ tients and other injury mechanisms in 25 patients. The median ISS (IQR) was 18 (9-27). Median systolic blood pressure on admission was 130 (range 120-140) and a median heart rate of 88 (range 76-100) beats per minute was encountered.

The Organ Injury Score of splenic injury was less than Grade IV in 66 patients, Grade IV in 11 and Grade V in 3 patients. No relevant comorbidities were found. Twenty patients had impaired mental status, of whom 2 patients were diagnosed with critical craniocerebral injuries (AIS 5) and 14 patients with AIS 3-4 craniocerebral injuries. Additionally, 4 patients had impaired GCSscores due to the trauma severity and hypovolemic shock state. One patient had a severe head injury diagnosed as well as a positive drug test on admission. In all other patients, intoxications were ruled out.

\section{Impact of neurological impairment on outcome of nonoperative management}

To determine the impact of neurological impairment we compared the patients with normal GCS-score and those with decreased GCS-scores. A total of 20 patients had diminished GCS. The median GCS-score (range) was 9 (6-12) and five patients had a GCSscore less than 7 .

The groups were similar in baseline patient characteristics and splenic injury scores. (Table 1). As anticipated, median ISS was significantly higher in the neurologically impaired group compared with group I (29 versus 17; $p=0.007)$. Hemoglobin levels were also slightly lower in the neurologically impaired group, however statistical significance was not reached $(8,4$ versus 7,$6 ; p=0.105)$. In both groups there was a male predominance.

Table 2 reviews outcome data for both neurologically impaired and non-impaired patients. Median length of stay ( 28 vs. 9 days; $p<0.001$ ) and duration of ICU stay (8 vs. 0 days; $p=0.005$ ) were significantly longer in patients with a decreased GCS-score. No differences in the failure rate of $\mathrm{NOM}$ and number of complications were found between the groups. In two patients, failure of NOM was prevented by angio-embolization. Failure of NOM did not occur in any patient with a contrast-blush on initial CT-scanning.

Among the 20 patients with neurological impairment, NOM was successful in 16 cases $(80 \%)$. In the group with regular trauma patients, NOM failed in 7 out of 62 individuals.

Failure of NOM occurred due to development of hemodynamic instability in seven patients, of whom 3 patients were neurologically impaired. Serum hemoglobin levels in these patients remained relatively unchanged over time. Nevertheless, surgical intervention was mandated in these patients due to the inadequate response to resuscitation and fluid challenge.

In three patients (including one neurologically impaired individual) a gradual progressive decrease in serum hemoglobin levels, combined with progressive sonographic intraabdominal fluid and tachycardia resulted in the need for surgical intervention. One patient with normal GCS-scores was operated on as he had gradually worsening hemodynamic parameters and deteriorating respiratory status.

Four spleen saving procedures were performed, of which two were in a patient with neurological impairment. The most frequent complications were pneumonia, extraabdominal abscesses, and ileus. Two patients developed ileus after laparotomy. One patient with impaired mental status required re-laparotomy, in which a hemicolectomy was performed to treat a mesenterial contusion with subsequent secondary ischemia. Re-laparotomy in this patient was indicated because of persistent fever combined with an ileus. There were slightly more complications encountered in neurologically impaired patients. However, this trend did not reach statistical significance and moreover none of the complications were associated with delayed diagnosis related to impaired mental status. No differences were seen between groups, regarding the severity and impact of diagnosed complications as measured by the Clavien-Dindosystem [22]. All complications are shown in Table 3. Mortality was not seen in patients selected for nonoperative management.

\section{Discussion}

There is clear evidence that supports non-operative management in blunt splenic injury $[6,9,11,15,23]$. However, controversy exists regarding the impact of altered mental 
Table 1 Patient and hemodynamic characteristics in the presence or absence of neurological impairment

\begin{tabular}{llll}
\hline & Group I: Normal mental status GCS $=14 / 15(n=62)$ & Group II: Altered mental status GCS $\leq 13(n=20)$ & $P$ value \\
\hline Age in years & $32(23-53)$ & $26(20-33)$ & 0.166 \\
Gender (M/F) & $48 / 14$ & $17 / 3$ & 0.545 \\
GCS & $15(15-15)$ & $9(6-12)$ & $P<0.001^{*}$ \\
AIS spleen & $2(2-3)$ & $2(2-3)$ & 0.290 \\
ISS & $17(4-24)$ & $29(18-34)$ & $0.007^{*}$ \\
Contrast blush on CT & 5 & 2 & 0.700 \\
Serum hemoglobin (mmol/l) & $8.4(7.8-8.9)$ & $7.6(7.1-8.4)$ & 0.105 \\
Systolic blood pressure (mmHg) & $130(120-145)$ & $125(113-140)$ & 0.727 \\
Pulse rate (BPM) & $88(77-100)$ & $90(70-103)$ & 0.845 \\
\hline
\end{tabular}

All data are in median (IQR): ${ }^{*}, p<0.05$ : Mann Whitney $\mathrm{U}$ test

status on the outcome and safety of NOM. The presence of concomitant head injury was considered a contraindication for non-operative therapy. There is concern that non-operative management in patients with altered mental status may miss early signs of intraabdominal hemorrhage that may subsequently delay surgical intervention [14-16]. We consider patients with impaired mental status as adequate candidates for non-operative management. Therefore, according to our hospital guidelines, patients with decreased GCS-scores have not been excluded from NOM during the past decade.
This study has shown that there are no differences in failure of NOM, complications due to NOM, or mortality between patients with normal mental status and those with impaired mental status. Thus, this study showed that hemodynamically stable patients with impaired mental status can safely be selected for NOM.

The findings of the current study are consistent with other series on intraabdominal solid organ injuries in which both spleen and liver injuries are analyzed [14, 24]. Archer et al. started a trend towards NOM in neurologically impaired patients in 1996. They showed, in a study with 187 observationally treated patients

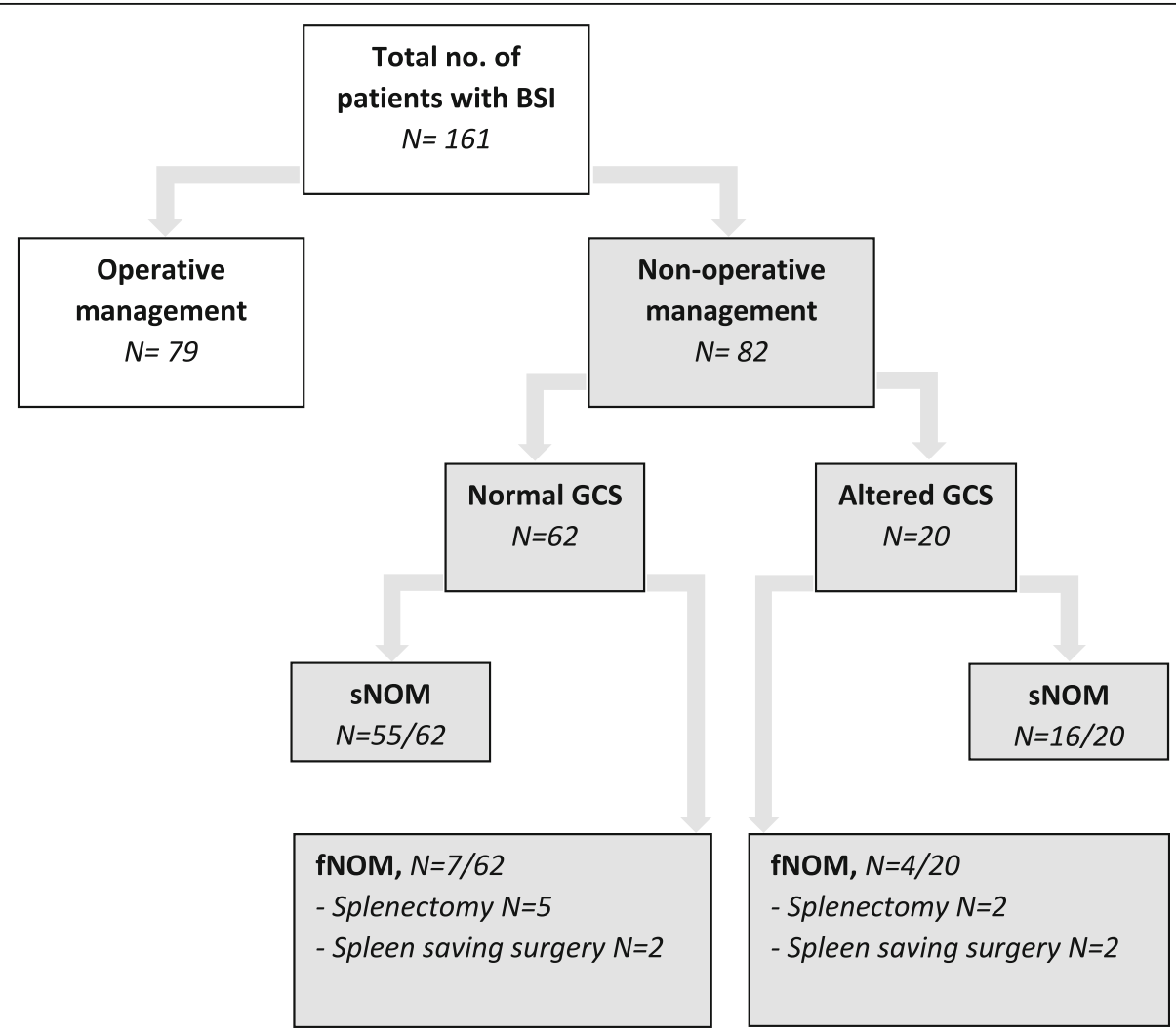

Fig. 1 Flowchart and patient selection 
Table 2 Outcome of nonoperative management in the presence or absence of neurological impairment

\begin{tabular}{llll}
\hline & Group I: Normal mental status GCS =14/15 $(\mathrm{n}=62)$ & Group II: Altered mental status GCS $\leq 13(\mathrm{n}=20)$ & $P$-value \\
\hline Failure of NOM & 7 & 4 & 0.449 \\
Splenectomy & $5 / 7$ & $2 / 4$ & 0.477 \\
ICU-stay (days) & $0(0-2)$ & $8(2-25)$ & $p<0.001^{*}$ \\
Length of hospital stay (days) & $9(7-19)$ & $28(13-59)$ & $0.005^{*}$ \\
Complications (no.) & 18 & 12 & 0.085 \\
Complication severity ${ }^{\mathrm{a}}$ & $0(0-2)$ & $2(0-2)$ & 0.069 \\
Mortality & 0 & 0 & $p>0.393$ \\
\hline
\end{tabular}

All data are in median (IQR); ${ }^{*}, p<0.05$ : Mann Whitney $\mathrm{U}$ test/Fisher Exact test. ${ }^{\mathrm{a}}$ According to Clavien-Dindo-classification

sustaining splenic and hepatic injury, no differences in morbidity or mortality between normal and neurologically impaired patients [24]. Furthermore, corresponding results were found by Keller et al. who studied pediatric patients with hepatic and splenic injuries [14]. These studies pooled splenic and hepatic injuries as they were based on the false assumption that splenic and hepatic injuries are comparable in treatment and clinical course. Our study exclusively valuated the impact of altered mental status on outcome of blunt splenic injury alone.

More recently, Shapiro et al. found in a study of 2327 patients sustaining kidney, liver or spleen injuries (AIS > 1 ), that nonoperative treatment was less likely to be initiated with worsening mental status, however, NOM in neurologically impaired patients sustaining liver, spleen or kidney injury was successful in more than $90 \%$ of cases [17].

Furthermore, the current study found similar success rates of NOM in both groups, despite higher injury severity scores in the neurologically impaired group versus the control group. This finding underlines the limited value of injury severity score calculations to determine therapy and to predict outcome of patients with blunt solid organ injuries.

Our findings are also in line with a study from Dhillon et al. on patients with both splenic and cerebral trauma selected for non-operative management.
Interestingly, they found higher success rates of NOM in patients with brain injury than those patients without brain injuries. They suggested that this may be a result of management aimed to prevent secondary brain injury [25]. Those patients with concurrent brain injuries require close monitoring and therefore obtain optimal hemodynamic therapy. The injured spleen is likely to benefit from optimized hemodynamic monitoring and management as well. This effect may have contributed to the relatively good outcome in our neurologically impaired patients as well.

It has been shown that hypotension, defined as a systolic blood pressure $<90 \mathrm{mmHg}$, is associated with impaired cerebral perfusion as well as with deprived outcome in patients with traumatic brain injury [26]. During the course of the current study, our resuscitation guidelines did not change and included a semirestrictive resuscitation protocol for all trauma cases, in which systolic blood pressure levels are maintained at a minimum of $90 \mathrm{mmHg}$. As other publications on permissive hypotension, utilize lower minimal systolic blood pressure levels (as low as $70 \mathrm{mmHg}$ [27]), than we do, we decided to define our protocol as semi-restrictive. In our view this approach can safely be applied to all trauma patients (including those with traumatic brain injury).

Table 3 Complications

\begin{tabular}{lll}
\hline & Group I: Normal mental status $(\mathrm{n}=62)$ & Group II: Impaired mental status $(\mathrm{n}=20)$ \\
\hline Pneumonia & 4 & 6 \\
Abscess (intra-abdominal) & 2 & 3 \\
Ileus & 4 & 0 \\
Respirarory failure & 3 & 0 \\
ARDS & 1 & 2 \\
Abscess (extra-abdominal) & 0 & 1 \\
Abdominal compartment syndrome & 1 & 0 \\
Pulmonary embolus & 1 & 0 \\
Sepsis & 2 & 0 \\
Total & 18 & 12 \\
\hline
\end{tabular}


The impact of hypertension on outcome is unclear und recommendations are controversial [28]. In our view, excessive use of catecholamines should be avoided in traumatic brain injury patients as this can lead to prominent variations in systolic blood pressure, which affects intracerebral capillary hydrostatic pressure and thereby may contribute to the development of cerebral edema [29-31].

Our study is limited by a relatively small sample size, although this sample size is comparable to other key publications in which data on hepatic and splenic injuries have been pooled [13]. Given the differences in therapy as well as prognosis and outcome between hepatic injuries and splenic injuries, we believe that there is a need to analyze outcome of splenic injury separately and this study is the first to do so. Furthermore, as we have used a strictly maintained prospective database, there are no missing data. Moreover, due to the detailed data provided by the database, precise information about the conditions surrounding complications, including the indications for laparotomy, were obtained. The relatively high rates of initial operative therapy and failure of NOM are most likely due to the relative trauma severity (and high trauma load) of patients admitted to our level one trauma center. This is supported by the high median ISS-score and large proportion of patients with highgrade splenic injury in the current study population.

\section{Conclusion}

The current study shows that non-operative management for blunt splenic trauma in patients with altered mental status is a viable treatment modality in wellequipped institutions. However, the presence of neurologic impairment is associated with prolonged ICU-stay and hospitalization, likely due to the management of the neural injury and related prolonged hemodynamic monitoring itself. We therefore recommend institutions with adequate monitoring facilities, to attempt nonoperative management for splenic injury in all hemodynamically stable patients without hollow organ injuries, regardless of neurological status.

\section{Abbreviations}

AIS: Abbreviated injury score; CT: Computed tomography; fNOM: Failure of nonoperative management; GCS: Glasgow Coma Scale; ICU: Intensive care unit; IQR: Interquartile range; ISS: Injury severity score; MOI: Mechanism of injury; NOM: Nonoperative management

\section{Acknowledgments}

Not applicable.

\section{Authors' contributions}

MT, TB, RP, HCP, LL designed the study. Data collection and screening was performed by MT, RS, TB, HT. Data interpretation and writing the manuscript was performed by MT, RS, HT. All authors read and approved the final manuscript.

\section{Funding}

No funding to be declared.

\section{Availability of data and materials}

The datasets analyzed during the current study are not publicly available due to privacy issues. Please contact author for data requests.

\section{Ethics approval and consent to participate}

The study was approved by the University Medical Center Utrechts ethical committee.

\section{Consent for publication}

Not applicable.

\section{Competing interests}

The authors declare that they have no competing interests.

Received: 10 May 2019 Accepted: 13 September 2019

Published online: 05 December 2019

\section{References}

1. Cox EF. Blunt abdominal trauma. A 5-year analysis of 870 patients requiring celiotomy. Ann Surg. 1984;199(4):467-74.

2. Davis JJ, Cohn I Jr, Nance FC. Diagnosis and management of blunt abdominal trauma. Ann Surg. 1976;183(6):672-8.

3. Leppäniemi A. Nonoperative management of solid abdominal organ injuries: from past to present. Scand J Surg. 2019. https://doi.org/10.1177/ 1457496919833220.

4. Peitzman AB, Richardson JD. Surgical treatment of injuries to the solid abdominal organs: a 50-year perspective from the journal of trauma. J Trauma. 2010;69(5):1011-21.

5. Chien-An L, Wu Y-T, Chien-Hung L, Shang-Yu W, Chih-Yuan F, Chi-Hsun H, et al. Hospital level variations in the trends and outcomes of the nonoperative management of splenic injuries - a nationwide cohort study. Scand J Trauma Resusc Emerg Med. 2019. https://doi.org/10.1186/s13049018-0578-y.

6. Bhangu A, Gupta A, Elvy A. Rates and predictors of surgery for patients with blunt splenic trauma in a district general hospital of the UK. Eur J Emerg Med. 2011;18(5):265-7.

7. Pachter HL, Guth AA, Hofstetter SR, Spencer FC. Changing patterns in the management of splenic trauma: the impact of nonoperative management. Ann Surg. 1998;227(5):708-17 discussion 717-9.

8. Bee TK, Croce MA, Miller PR, Pritchard FE, Fabian TC. Failures of splenic nonoperative management: is the glass half empty or half full? J Trauma. 2001:50(2):230-6

9. Nix JA, Costanza M, Daley BJ, Powell MA, Enderson BL. Outcome of the current management of splenic injuries. J Trauma. 2001;50(5):835-42.

10. Cobum MC, Pfeifer J, DeLuca FG. Nonoperative management of splenic and hepatic trauma in the multiply injured pediatric and adolescent patient. Arch Surg. 1995;130(3):332-8.

11. McIntyre LK, Schiff M, Jurkovich GJ. Failure of nonoperative management of splenic injuries: causes and consequences. Arch Surg. 2005;140(6):563-8 discussion 568-9.

12. Rose J, Valtonen $\mathrm{S}$, Jennett B. Avoidable factors contributing to death after head injury. Br Med J. 1977;2(6087):615-8.

13. Gurdjian ES. Acute head injuries. Surg Gynecol Obstet. 1978;146(5):805-20.

14. Keller MS, Sartorelli KH, Vane DW. Associated head injury should not prevent nonoperative management of spleen or liver injury in children. J Trauma. 1996;41(3):471-5.

15. Cogbill TH, Moore EE, Jurkovich GJ, Morris JA, Mucha P Jr, Shackford SR, et al. Nonoperative management ofblunt splenic trauma: a multicenter experience. J Trauma. 1989;29(10):1312-7.

16. Koury HI, Peschiera JL, Welling RE. Non-operative management of blunt splenic trauma: a 10-year experience. Injury. 1991;22(5):349-52.

17. Shapiro MB, Nance ML, Schiller HJ, Hoff WS, Kauder DR, Schwab CW. Nonoperative management of solid abdominal organ injuries from blunt trauma: impact of neurologic impairment. Am Surg. 2001;67(8):793-6.

18. Olthof DC, Joosse P, van der Vlies CH, de Haan RJ, Goslings JC. Prognostic factors for failure of nonoperative management in adults with blunt splenic injury: a systematic review. J Trauma. 2013;74(2):546-57. 
19. Coccolini F, Montori G, Catena F, Kluger Y, Biffle W, Moore EE. Splenic trauma: WSES classification and guidelines for adult and pediatric patients. World J Emerg Surg. 2017;18(12):40. https://doi.org/10.1186/s13017-0170151-4.

20. Gennarelli TA, Wodzin E. The Abbreviated Injury Scale 2005. Update 2008. Des Plaines: American Association for Automotive Medicine (AAAM); 2008.

21. Olthof DC, Joosse P, Bossuyt PM, de Rooij PP, Leenen LP, Wendt KW, Bloemers FW, Goslings JC. Observation versus embolization in patients with blunt splenic injury after trauma: a prospensity score analysis. World J Surg. 2016;40(5):1264-71.

22. Dindo D, Demartines N, Clavien PA. Classification of surgical complications. Ann Surg. 2004;240(2):205-13.

23. Peitzman AB, Heil B, Rivera L, Federle MB, Harbrecht BG, Clancy KD, et al. Blunt splenic injury in adults: multi institutional study of the eastern Association for the Surgery of trauma. J Trauma. 2000:49(2):177-87 discussion 187-9.

24. Archer LP, Rogers FB, Shackford SR. Selective nonoperative management of liver and spleen injuries in neurologically impaired adult patients. Arch Surg. 1996;131(3):309-15.

25. Dhillon NK, Barmparas G, Thomsen GM, Patel KA, Linaval NT, Gillette E, et al. Nonoperative management of blunt splenic trauma in patients with traumatic brain injury: feasibility and outcomes. World J Surg. 2018:42(8): 2404-11.

26. Spaite DW, Hu C, Bobrow BJ, Chikani V, Barnhart B, Gaither JB, et al. Association of out-of-hospital hypotension depth and duration with traumatic brain injury mortality. Ann Emerg Med. 2017:70(4):522-30.

27. Dutton R, Mackenzie C, Scalea T. Hypotensive resuscitation during active haemorrhage: impact on in-hospital mortality. J Trauma. 2002:52(6):1141-6.

28. Caplan HW, Cox CS. Resuscitation strategies for traumatic brain injury. Curr Surg Rep. 2019;7(7). https://doi.org/10.1007/s40137-019-0237-x.

29. Graende PO. Critical evaluation of the Lund concept for treatment of severe traumatic head injury, 25 years after its introduction. Front Neurol. 2017;8: 315.

30. Nordstroem CH, Koskinen LO, Olivecrona M. Aspects on the physiological and biomechanical foundations of neurocritical care. Front Neurol. 2017:8: 274

31. Krishnamoorthy C, Chaikittisilpa N, Kiachai T, Vavilala M. Hypertension after severe traumatic brain injury: friend or foe? J Neurosug Anesthesiol. 2017; 29(4):382-7.

\section{Publisher's Note}

Springer Nature remains neutral with regard to jurisdictional claims in published maps and institutional affiliations.

Ready to submit your research? Choose BMC and benefit from:

- fast, convenient online submission

- thorough peer review by experienced researchers in your field

- rapid publication on acceptance

- support for research data, including large and complex data types

- gold Open Access which fosters wider collaboration and increased citations

- maximum visibility for your research: over $100 \mathrm{M}$ website views per year

At $\mathrm{BMC}$, research is always in progress.

Learn more biomedcentral.com/submissions 